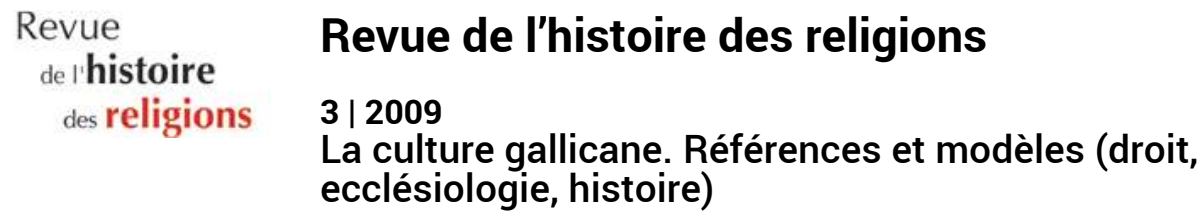

La culture gallicane. Références et modèles (droit, ecclésiologie, histoire)

\title{
Louis Ellies Du Pin (1657-1719), historien de Byzance
}

Louis Ellies Du Pin (1657-1719), a Historian of Byzantium

Marie-Hélène Blanchet

\section{(2) OpenEdition}

\section{Journals}

Édition électronique

URL : http://journals.openedition.org/rhr/7270

DOI : $10.4000 /$ rhr.7270

ISSN : 2105-2573

Éditeur

Armand Colin

\section{Édition imprimée}

Date de publication : 1 juillet 2009

Pagination : 413-428

ISBN : 978-2200-92591-8

ISSN : 0035-1423

Référence électronique

Marie-Hélène Blanchet, «Louis Ellies Du Pin (1657-1719), historien de Byzance », Revue de l'histoire des religions [En ligne], 3 | 2009, mis en ligne le 01 juillet 2012, consulté le 19 avril 2019. URL : http:// journals.openedition.org/rhr/7270 ; DOI : 10.4000/rhr.7270 


\section{Louis Ellies Du Pin (1657-1719), historien de Byzance}

Louis Ellies Du Pin (1657-1719) est l'un des rares auteurs français de la fin $d u X_{X I I}^{e}$ siècle à proposer une vision relativement impartiale de l'histoire byzantine: il relate de manière nuancée les principaux affrontements qui ont opposé l'Église byzantine et la papauté entre le vII et le $X V^{e}$ siècle. Il semble que son absence de préjugés et son intérêt pour l'orthodoxie médiévale puisse en partie être mis sur le compte de sa sensibilité gallicane.

\section{Louis Ellies Du Pin (1657-1719), a Historian of Byzantium}

Louis Ellies Du Pin (1657-1719) is one of the few French authors of the late seventeenth century to offer a relatively unbiased view of Byzantine history: he recounts with subtlety the major clashes between the Byzantine Church and the papacy from the seventh to the fifteenth century. It seems that his lack of prejudice and his interest in Medieval orthodoxy may partly be on account of his Gallican sensibility. 
Il peut paraître étrange, à première vue, d'évoquer Byzance comme l'un des modèles de la culture gallicane. On connaît certes l'importance qu'ont accordée à l'histoire les érudits français des $\mathrm{XVI}^{\mathrm{e}}$ et $\mathrm{XVII}{ }^{\mathrm{e}}$ siècles; cependant leurs sujets de prédilection, loin de les conduire vers le Moyen Âge grec, les ont plutôt portés vers l'étude des origines de la nation française ou vers les premiers temps du christianisme. De la même façon, l'intérêt humaniste pour les œuvres classiques, qui a permis de remettre en lumière certains auteurs grecs, est resté cantonné aux productions antiques. Ainsi la civilisation byzantine semble à tous égards éloignée des préoccupations savantes de l'époque moderne.

À bien examiner les choses, ce jugement est pourtant faux : l'Empire byzantin connait en effet une certaine vogue au XVII ${ }^{\mathrm{e}}$ siècle en France. En témoigne l'importante production imprimée qu'il inspire, depuis la tragédie de Corneille Héraclius, empereur d'Orient, jusqu'à des ouvrages de pure érudition, en passant par la publication de la «Byzantine du Louvre », grande entreprise d'édition de textes grecs médiévaux. Cet intérêt pour le monde byzantin n'est pas dénué de paradoxes. La période protobyzantine, de Constantin à Justinien, trouve évidemment grâce aux yeux des spécialistes de l'Empire romain et des débuts du christianisme: l'époque des premiers conciles constitue en effet la référence par excellence de tous ceux qui s'attachent à l'histoire ecclésiastique, tel Le Nain de Tillemont par exemple. Il n'en va pas de même de la période postérieure : à partir de la querelle iconoclaste, et plus encore lorsque le schisme vient séparer définitivement les deux moitiés de la chrétienté, Byzance devient pour la plupart des catholiques un véritable repoussoir. Rares sont donc les auteurs français qui s'intéressent réellement à l'histoire religieuse byzantine au-delà du $\mathrm{vII}^{\mathrm{e}}$ siècle : la tradition orthodoxe n'est bien souvent invoquée qu'à titre de comparaison avec les pratiques protestantes, dans un esprit tout à fait polémique.

Parmi les rares auteurs du XVII ${ }^{\mathrm{e}}$ siècle qui consacrent quelques développements à l'histoire méso-byzantine se trouve le théologien gallican Louis Ellies Du Pin (1657-1719) ${ }^{1}$. Ce professeur de philo-

1. Sur Du Pin, voir Yves Poutet, « Du Pin, Louis Ellies », dans le Dictionnaire de biographie française, XII, Paris, 1970, col. 366-368; Jacques Grès-Gayer, « Un 
sophie du Collège royal est l'auteur de la Nouvelle bibliothèque des auteurs ecclésiastiques, en dix-neuf tomes, consacrée aux auteurs chrétiens à la fois latins et grecs depuis le $\mathrm{I}^{\mathrm{er}}$ siècle jusqu'à l'époque moderne ${ }^{2}$. À cette vaste entreprise éditoriale appartient l'Histoire des controverses et des matières ecclésiastiques, qui porte précisément sur l'histoire religieuse de l'Occident et de l'Orient chrétien entre le $\mathrm{IX}^{\mathrm{e}}$ et le $\mathrm{XV}^{\mathrm{e}}$ siècle et à laquelle est consacrée pour l'essentiel cette étude ${ }^{3}$. Non seulement l'œuvre de Du Pin apparaît comme originale en raison de son ampleur exceptionnelle, mais la façon dont l'auteur aborde l'histoire byzantine contraste aussi avec ce qu'écrivent ses contemporains sur le sujet. Nous tenterons de montrer en quoi cette vision de Byzance peut être considérée comme spécifique, et nous proposerons quelques pistes permettant peut-être de lier cette singularité d'historien aux convictions gallicanes de son auteur.

\section{Du Pin et les études byzantines en France À la fin du XVII ${ }^{\mathrm{E}}$ Siècle}

$\mathrm{Au}$ moment où il rédige son Histoire des controverses consacrée à la période médiévale, c'est-à-dire dans les années 1690, Louis Ellies Du Pin peut s'appuyer sur les travaux déjà poussés de ses prédécesseurs ${ }^{4}$. Il ne manque aucune occasion de s'y référer, mais il conçoit l'œuvre qu'il entreprend comme un projet nouveau, beaucoup plus complet que tout ce qui a été réalisé jusqu'alors. Pas plus que ses contemporains, Du Pin n'est motivé par une quelconque sympathie pour ces lointains frères chrétiens que sont les

théologien gallican, témoin de son temps : Louis Ellies Du Pin (1657-1719) », Revue d'histoire de l'Église de France, 72, 1986, p. 67-121; Id., « Le gallicanisme de Louis Ellies Du Pin (1657-1719)», Lias, n 18, 1991, p. 37-81.

2. Louis Ellies Du Pin, Nouvelle bibliothèque des auteurs ecclésiastiques, Paris, 1693-1715.

3. Id., Histoire des controverses et des matières ecclésiastiques traitées dans le neuviéme[-quinziéme] siécle, Paris, 1694-1698.

4. Outre la "Byzantine du Louvre» ou Corpus Byzantinae historiae, qui comprend vingt-sept volumes parus à Paris entre 1645 et 1711 , Du Pin avait à sa disposition les traductions latines de François Combefis, Bibliotheca Patrum concionatoria, Paris, 1662 (8 vol.), ainsi que celles de Jean-Baptiste Cotelier, Ecclesiae Graecae monumenta, Paris, 1677-1692 (4 vol.). Sur les débuts de l'historiographie byzantine, voir Agostino Pertusi, Storiografia umanistica e mondo bizantino, Palerme, 1967. Sur l'érudition française au XVII ${ }^{\mathrm{e}}$ siècle, voir surtout Bruno Neveu, Érudition et religion aux XVII et XVIII siècles, Paris, 1994. 
orthodoxes : il les considère en effet avec une profonde condescendance du fait de leur inculture. Évoquant les auteurs grecs du $\mathrm{XV}^{\mathrm{e}}$ siècle, il écrit :

« Ceux qui ont vécu dans le Siécle dont nous parlons, ont encore de bons restes; mais ceux qui sont venus depuis, ont toûjours degeneré, \& sont enfin tombés dans l'ignorance, pour ne pas dire dans la stupidité où nous les voïons presentement. Ainsi les Auteurs dont nous allons parler peuvent être considerés comme les derniers Grecs ${ }^{5}$. »

Seuls les Byzantins d'antan peuvent donc éveiller en lui un intérêt intellectuel, dans la mesure où ils se présentent comme les descendants à la fois des Grecs anciens et des Pères orientaux, mais leurs héritiers ne méritent certes pas la moindre attention aux yeux de Du Pin. Notre auteur ne se singularise donc en rien quant à son jugement sur les orthodoxes de son époque : comme les autres érudits français, il affiche une profonde indifférence à l'égard de toute préoccupation missionnaire.

Quelle est donc la raison qui le pousse à inclure Byzance dans son projet? Comme il l'écrit dans la préface du premier volume de la Nouvelle Bibliothèque, Du Pin a l'ambition de faire l'œuvre la plus exhaustive possible : il relève les insuffisances de ses prédécesseurs, notamment Baronius, Bellarmin et Labbe ${ }^{6}$, qui ont composé des ouvrages du même genre, selon lui incomplets et parfois même erronés, en particulier sur le plan de la critique d'attribution des œuvres. Il compte pour sa part donner un aperçu des écrits de tous les auteurs ecclésiastiques, occidentaux et orientaux, jusqu'à son époque, ce qu'il a effectivement réussi à réaliser entre 1686 et 1714. Sa Bibliothèque est conçue en réalité comme un instrument de travail organisé selon un plan chronologique, à vocation encyclopédique. Il énumère dans la seconde partie de sa préface les différentes rubriques qu'il s'attache à remplir de façon systématique : pour tous les auteurs, il commence par une rapide biographie et s'autorise à cette occasion quelques développements sur la

5. Louis Ellies Du Pin, Histoire des controverses et des matières ecclésiastiques traitées dans le quinziéme siécle, Paris, 1698, p. 409.

6. Voir Id., Nouvelle bibliothèque des auteurs ecclésiastiques, I, Des auteurs des trois premiers siecles de l'Eglise, Paris, 1686, p. 4-6. Les ouvrages auxquels se réfère Du Pin sont les suivants : César Baronius, Annales ecclesiastici, Rome, 1588-1607; Robert Bellarmin, De Scriptoribus ecclesiasticis liber unus, Lyon, 1613 ; Philippe Labbe, De scriptoribus ecclesiasticis, quos attigit Eminentiss. S. R. E. Card. Robertus Bellarminus, Philologica et historica dissertatio, Paris, 1660. 
période concernée 7 . Notons d'emblée que l'histoire n'est donc pas l'objet principal de Du Pin : il prétend ne l'aborder que pour éclairer la pensée des auteurs qu'il présente. En réalité pourtant, dans les volumes qu'il consacre au Moyen Âge, le récit historique, tout en étant resserré et dense, occupe une place importante en tête de chaque chapitre. Après cette entrée en matière, Du Pin dresse un catalogue critique des auteurs et de leurs œuvres - y compris les œuvres disparues, ce dont il est particulièrement fier -; il en résume «l'argument », à savoir les idées principales, puis en signale toutes les éditions connues. Du Pin revendique une approche synthétique : il ne cherche pas à entrer dans le détail des doctrines présentées, par crainte, explique-t-il, d'ennuyer son lecteur'. Il émet pour finir un jugement personnel sur le style et la valeur des auteurs étudiés, seul moment où il se départit de la forme strictement informative à laquelle il s'astreint en général. L'adjonction de tables et d'index à la fin de chaque volume ajoute au caractère pratique de l'œuvre, destinée non à être lue en continu, mais à être consultée comme un dictionnaire.

Byzance trouve tout naturellement sa place dans ce projet ambitieux. Du Pin ne semble pas s'être particulièrement interrogé sur la nécessité d'inclure les auteurs byzantins au même titre que les Pères grecs : certes, Baronius éditait déjà des documents émanant des empereurs byzantins et des patriarches lorsqu'il en disposait; à leur tour, Robert Bellarmin et Philippe Labbe, que Du Pin cite comme ses prédécesseurs directs, mentionnaient les principaux auteurs grecs du Moyen Âge, mais ils ne fournissaient que des notices succinctes, n'évoquant que les écrits strictement ecclésiastiques et passant sous silence une grande partie des chroniques9. Son souci d'exhaustivité poussait sans aucun doute Du Pin à une approche plus complète de la littérature byzantine; mais faut-il aussi voir dans son choix

7. Louis Ellies Du Pin, Nouvelle bibliothèque des auteurs ecclésiastiques, I, Des auteurs des trois premiers siecles de l'Eglise, Paris, 1686, p. 15 : «Car c'est une des choses qui sert le plus à nous faire comprendre la pensée d'un Auteur, c'est de sçavoir dans quel temps \& dans quel esprit il écrivoit, quels Heretiques il combattoit, quel dogme il vouloit établir, en quel état \& en quelle disposition il étoit pour lors " .

8. Ibid., p. 20 : « Je l'ai aussi rendu le moins ennuieux qu'il m'a été possible, en ne gardant pas toûjours une methode exacte $\&$ en ne faisant pas une analise scrupuleuse des propositions \& des raisonnemens ».

9. Voir supra, note 6. Bellarmin et Labbe mentionnent certains auteurs ecclésiastiques byzantins, par exemple Jean Damascène ou Photios. 
une décision personnelle, suscitée par un goût particulier pour les sources grecques médiévales? Du Pin cite en effet comme l'un de ses modèles le patriarche orthodoxe du $\mathrm{Ix}^{\mathrm{e}}$ siècle Photios, auteur en son temps d'une immense Bibliothèque dont Du Pin se montre très admiratif :

«Il n'y a pas un de tous les ouvrages dont nous venons de parler qui comprenne tout ce qu'on peut dire sur les Auteurs ecclesiastiques. [...] Il n'y a que Photius parmi les anciens qui fasse l'abregé, \& qui porte son jugement des Livres dont il parle, mais il n'a travaillé que sur fort peu d'Auteurs ecclesiastiques ${ }^{10}$. »

Se plaçant sous de tels auspices, Du Pin élargit donc le spectre des auteurs et des textes dont il donne un aperçu, et il profite pour ce faire de tout le travail d'édition des sources grecques qui a eu lieu avant lui durant un demi-siècle. Mais il va encore plus loin: alors que tel n'était pas du tout son projet initial, Du Pin est conduit à fournir une trame générale de l'histoire religieuse byzantine, afin de replacer dans leur contexte les auteurs qu'il mentionne, et il est en France l'un des premiers à le faire. Du Pin devient donc historien de Byzance inconsciemment, presque à son corps défendant : cette part de son œuvre qui, à ses propres yeux, est relativement accessoire, apparaît aujourd'hui comme un récit fidèle et cohérent.

\section{UN TRAITEMENT DE L'HISTOIRE ORIGINAL ET NUANCÉ}

Du Pin ne s'attache pas avec la même précision à tous les aspects de l'histoire de l'orthodoxie médiévale. Il privilégie à l'évidence les épisodes où les Byzantins se trouvent en relation avec l'Occident, relations la plupart du temps conflictuelles. C'est ainsi qu'il développe cinq événements auxquels il accorde une réelle importance : la querelle iconoclaste des $\mathrm{VIII}^{\mathrm{e}}$ et $\mathrm{IX}^{\mathrm{e}}$ siècles, le schisme de Photios à la fin $\mathrm{du}_{\mathrm{IX}}^{\mathrm{e}}$ siècle, puis le schisme définitif de 1054, et enfin les deux tentatives de réunion des Églises lors des conciles de Lyon II, en 1274, et de Florence, en 1438-1439. À chaque fois, l'Église byzantine se heurte à la Papauté, et Du Pin relate l'affrontement sans prendre parti, au moins en apparence.

10. Louis Ellies Du Pin, Nouvelle bibliothèque des auteurs ecclésiastiques, I, Des auteurs des trois premiers siecles de l'Eglise, Paris, 1686, p. 9. 
Avant d'analyser la manière dont il rend compte de ces crises, il n'est pas inutile de s'attarder d'abord quelques instants sur ce dont il ne parle pas. Les croisades constituent l'un des principaux événements médiévaux auxquels sont mêlés tant l'Église romaine que les Byzantins : or Du Pin garde, semble-t-il, un silence assez pudique sur le sujet. C'est particulièrement clair à propos de la quatrième croisade, détournée en 1204 vers Constantinople : Du Pin tend à imputer la première chute de l'Empire byzantin à des querelles intestines plutôt qu'au désir de conquête des croisés, se gardant d'en fournir la moindre explication et n'émettant aucun jugement ${ }^{11}$. Il poursuit son récit comme si de rien n'était, évoquant ensuite avec beaucoup de naturel «le nouvel Empereur Baudoüin », alors que l'Empire byzantin se trouve désormais sous la domination des Latins. Lorsqu'il traite un peu plus loin du règne de Michel VIII Paléologue, l'empereur byzantin qui a reconquis Constantinople en 1261, Du Pin lance de façon assez inopinée, mais cette fois très critique, une dernière allusion au phénomène de la croisade :

« Michel Paleologue prévoïant que les Papes ne manqueroient pas d'armer contre lui les Princes d'Occident pour reprendre Constantinople, se resolut de proposer la réunion des Grecs avec l'Eglise romaine, afin de se délivrer par-là de la crainte de ses terribles Croisades ${ }^{12}$. »

Du Pin restitue ainsi fugitivement le point de vue des Byzantins sur les expéditions militaires chrétiennes, mais évite dans l'ensemble très nettement ce sujet ${ }^{13}$.

S'il reste discret sur les croisades, Du Pin analyse de façon beaucoup plus libre certains autres différends mettant aux prises

11. Voir Id., Histoire des controverses et des matières ecclésiastiques traitées dans le treiziéme siécle, Paris, 1698, p. 299-300.

12. Ibid., p. 305.

13. Peut-être Du Pin songe-t-il ici à ménager la susceptibilité de Louis XIV, qui cherche alors à prouver ses droits historiques sur l'ancien Empire byzantin en s'appuyant précisément sur le précédent que constitue la conquête et l'établissement de la domination latine sur Constantinople en 1204. En témoignent les premiers mots de la préface de Du Cange dans son édition de la chronique de Villehardouin : «Sire, ie ne presente pas à Vostre Maiesté des terres étrangeres, \& de nouveaux mondes, quand ie luy offre l'Empire de Constantinople, puisque c'est un Trône sur lequel la valeur \& la vertu ont élevé vos Ayeux » (Charles Du Fresne Du Cange, Histoire de l'empire de Constantinople sous les empereurs françois, Paris, 1657). Voir aussi Byzance retrouvée. Érudits et voyageurs français, XVI'XVIII ${ }^{e}$ siècles, éd. Marie-France Auzépy et Jean-Pierre Grélois, Paris, 2001, p. 18, 39 et 86. 
les Byzantins et la papauté. C'est ainsi qu'il retrace en détail la querelle iconoclaste lorsqu'il présente le contexte historique des $\mathrm{VII}^{\mathrm{e}}$ et $\mathrm{VIII}^{\mathrm{e}}$ siècles. Ses conclusions à propos du culte des images témoignent d'une juste compréhension de la place de l'icône dans le monde orthodoxe et de la théologie byzantine de l'image :

«Pour ce qui est du culte qu'on leur a rendu, il est certain qu'il ne se peut point rapporter aux Images, \& qu'on n'a aucune veneration pour la matiere dont elles sont composées, non plus que pour leur forme \& pour leur figure : mais seulement qu'à leur présence on donne des marques extérieures de veneration qu'on a pour ce qu'elles représentent. Ce culte ainsi expliqué, comme il l'a été par la pluspart des défenseurs des Images, ne peut point être accusé ni taxé d'idolatrie : ceux même chez lesquels il n'étoit pas en usage n'en disconviennent $\operatorname{pas}^{14} . »$

Mais Du Pin est tout aussi exact lorsqu'il évoque les excès des iconodoules - les partisans des images - qui ont été à l'origine de la réaction contraire. Il présente ainsi Michel II (820-829), l'un des empereurs iconoclastes, comme un modéré qui impose seulement quelques limites à des pratiques populaires déviantes :

« [Il fit faire un] Réglement par lequel il défendoit des abus qu'il prétendoit s'être glissez dans le culte des images; comme d'ôter les Croix des Eglises pour y mettre des Images, d'adorer les Images mêmes; de mettre des habits à des Statuës, \& de les faire servir de maraines aux enfans dans l'administration du Baptême $[\ldots]^{15}$. »

Ainsi Du Pin n'aborde pas la question de manière réductrice, en stigmatisant d'emblée les iconoclastes comme de mauvais chrétiens ${ }^{16}$; il est au contraire sensible à leurs arguments et au problème que pose en soi la représentation du sacré.

14. Louis Ellies Du Pin, Nouvelle bibliothèque des auteurs ecclésiastiques, V, Des auteurs du septiéme \& du huitiéme siécle de l'Eglise, Paris, 1691, p. 494.

15. Id., Histoire des controverses et des matières ecclésiastiques traitées dans le neuviéme siécle, Paris, 1694, p. 2. Ces détails sont effectivement connus par la lettre de Michel II à Louis le Pieux du 10 avril 824, qui était publiée dans les Annales ecclésiastiques de Baronius (Annales ecclesiastici auctore Caesare Baronio, XIV, Lucques, 1743, p. 62-66). La pratique consistant à donner des icônes pour parrain ou marraine à un enfant lors de son baptême est aussi attestée dans une lettre de Théodore Stoudite: voir Theodori Studitae epistulae, éd. Georgios Fatouros, Berlin, 1992, I, lettre 17, p. $48^{4-6}$ et p. $158^{*}$.

16. C'est en revanche ce que fait l'ancien jésuite Louis Maimbourg (16101686), qui dresse un parallèle entre les iconoclastes byzantins et les protestants : voir Louis Maimbourg, Histoire de l'hérésie des iconoclastes et de la translation de l'Empire aux François, Paris, 1674. 
Dans ce cas comme dans d'autres, la sensibilité de Du Pin à l'égard de points de vue non conformes à la doctrine officielle de l'Église ne tient pas au hasard : elle provient du désir de justifier et, si possible, de faire prévaloir la position singulière qu'a tenue l'Église de France sur ces mêmes questions. Ainsi, lorsqu'il traite de la controverse sur les images, Du Pin a constamment à l'esprit les difficultés qu'ont suscitées les décisions du concile de Nicée II en Occident et le refus qu'y a opposé Charlemagne. Dans le volume de la Nouvelle Bibliothèque consacré aux auteurs des VII et VIII ${ }^{\mathrm{e}}$ siècles, $\mathrm{Du}$ Pin présente la querelle iconoclaste selon le plan suivant ${ }^{17}$ : il commence par exposer en une dizaine de pages l'enchaînement des faits à Byzance jusqu'au concile de Nicée en 787 ; puis il consacre vingt-cinq pages à l'analyse des actes du concile, tout en introduisant des commentaires critiques sur les arguments scripturaires et patristiques invoqués par les partisans des images; il poursuit pendant plus de quinze pages en s'attachant à la rédaction des Libri Carolini, commandés par Charlemagne à Théodulf en prévision du concile de Francfort de 794. Du Pin exprime donc des sympathies iconoclastes évidentes, qui se retrouvent dans son jugement sur Michel II déjà cité; mais si Du Pin est si élogieux envers cet empereur iconoclaste, c'est aussi parce que Michel II est allé jusqu'à solliciter l'intervention de Louis le Pieux dans la querelle et s'est montré prêt à «[suivre] le sentiment de l'Eglise de France $»^{18}$. Une telle interprétation est certes biaisée et manifestement francocentrée; cependant cette optique permet à Du Pin d'éviter toute caricature à propos des iconoclastes byzantins.

Pour les mêmes raisons, Du Pin livre un récit dans l'ensemble très équilibré des conflits qui ont opposé les Byzantins à la papauté à partir du $\mathrm{IX}^{\mathrm{e}}$ siècle : ses propres réticences à l'égard des excès de la puissance pontificale le conduisent tout naturellement à comprendre le point de vue grec. Les exemples sont ici très nombreux : nous n'en présenterons que deux. On se souvient que Du Pin plaçait son œuvre sous le patronage de Photios, le patriarche de Constantinople de la seconde moitié du IX ${ }^{\mathrm{e}}$ siècle; or celui-ci n'était pas le Byzantin le plus recommandable : il avait été au centre du premier grave

17. Louis Ellies Du Pin, Nouvelle bibliothèque des auteurs ecclésiastiques, V, Des auteurs du septiéme \& du huitiéme siécle de l'Eglise, Paris, 1691, p. 436483.

18. Ibid., p. 485. 
affrontement entre les Églises romaine et grecque, puisqu'il avait osé excommunier le pape Nicolas ${ }^{\text {er }}$ en 867 , après avoir été luimême déposé par le souverain pontife lors du synode du Latran en 863. Ces événements sont relatés dans le détail par Du Pin, de manière relativement neutre; c'est surtout le récit de la fin de la crise qui attire l'attention. Après diverses péripéties, dont le concile anti-photien de 869-870 à Constantinople, Photios est finalement rétabli en 878 sur le trône patriarcal par l'empereur Basile I ${ }^{\text {er }}$, qui cherche à faire reconnaître cette élection par le pape Jean VIII, alors que les relations entre les deux Églises sont totalement rompues. Pour convaincre le souverain pontife, Basile $\mathrm{I}^{\mathrm{er}}$ lui offre son aide militaire contre les incursions arabes et accepte surtout de laisser à Rome la juridiction sur la Bulgarie, importante terre de mission. D'après Du Pin, c'est ce dernier argument qui décide Jean VIII, soit des considérations de pure politique religieuse :

«L'Empereur Grec dont les forces commençoient à se rétablir en Italie, lui promettoit de délivrer les côtes de la Campanie des courses des Sarrazins, \& de lui rendre la Bulgarie. On alléguoit pour motif du rétablissement de Photius, le bien de la paix, \& la réünion des esprits. [...] [Jean VIII] ajoûtoit enfin, qu'il n'accordoit tout ceci qu'à condition qu'on lui cederoit la Bulgarie, \& que le Patriarche de Constantinople n'y prétendroit avoir aucun droit $^{19}$. »

Du Pin souligne ici la confusion entre pouvoir spirituel et pouvoir temporel, le pape étant prêt à transiger sur des questions de fond s'il accroît l'étendue de sa juridiction. De fait, peu après, en 879-880, se tient à Constantinople le concile qui marque la fin du schisme et où est reconnue en théorie l'autorité de Rome sur tout l'Illyricum occidental. Notons que l'historiographie moderne, comme Du Pin, souligne l'enjeu que représentait alors pour les deux Églises la christianisation des Slaves : il est exact que la question de la Bulgarie était alors au centre de la querelle entre Rome et Constantinople ${ }^{20}$.

Le regard que Du Pin porte sur les différents papes est encore plus critique lorsqu'il relate leur attitude à l'égard des Grecs à l'occasion des conciles: les deux tentatives d'Union des Églises romaine et orthodoxe de 1274 et de 1439 se sont soldées par des

19. Louis Ellies Du Pin, Histoire des controverses et des matières ecclésiastiques traitées dans le neuviéme siécle, Paris, 1694, p. 330. 1950.

20. Voir François Dvornik, Le schisme de Photius. Histoire et légende, Paris, 
échecs, et Du Pin n'est pas loin d'imputer à l'intransigeance et à la maladresse des souverains pontifes un tel résultat. À cet égard, le déroulement du concile de Florence fournit plusieurs épisodes au cours desquels se manifeste l'arrogance du pape Eugène IV : c'est le cas par exemple lors de l'arrivée des représentants de l'Église orthodoxe à Ferrare en mars 1438, Eugène IV exigeant que tous les métropolites orthodoxes, jusqu'au patriarche lui-même, viennent lui baiser les pieds :

«Il y eut d'abord des difficultés sur la manière dont le Patriarche, \& les Evêques Grecs aborderoient le Pape; il vouloit exiger d'eux qu'ils se prosternassent pour lui baiser les pieds : mais ils le refusèrent avec tant de fermeté, que le Pape fut obligé de se relâcher de sa prétention, $\&$ de les recevoir sans cette cérémonie ${ }^{21}$. »

Ce détail est tiré de la chronique d'un Byzantin hostile à l'Union, Sylvestre Syropoulos, qui raconte à sa manière le concile de Florence : comme Syropoulos est le seul auteur à évoquer cet incident, c'est nécessairement de son récit que Du Pin s'est servi ${ }^{22}$. Il n'est pas rare que Du Pin reprenne ainsi fidèlement sa source grecque, ce qui confère à sa présentation des événements un caractère équilibré qu'on ne retrouve pas ailleurs ${ }^{23}$. Dans son récit du concile florentin, Du Pin mentionne aussi l'insistance du pape sur les privilèges qui lui reviennent eu égard à la primauté du siège de Rome; mais il raconte aussi, avec une certaine ironie, le coup de force d'Eugène IV, alors que le décret d'Union est en cours de rédaction en juin 1439:

21. Louis Ellies Du Pin, Histoire des controverses et des matières ecclésiastiques traitées dans le quinziéme siécle, Paris, 1698, p. 136.

22. Voir Les "Mémoires" du Grand Ecclésiarque de l'Église de Constantinople, Sylvestre Syropoulos, sur le concile de Florence (1438-1439), éd. et trad. Vitalien Laurent, Rome-Paris, 1971, p. 230-235.

23. La manière positive dont Du Pin présente la chronique de Syropoulos contraste par exemple avec le jugement émis par le jésuite Philippe Labbe. Selon Du Pin, «elle est écrite en forme de Memoires : mais avec beaucoup de netteté, \& même de pureté » (Louis Ellies Du Pin, Histoire des controverses et des matières ecclésiastiques traitées dans le quinziéme siécle, Paris, 1698, p. 414). Selon Philippe Labbe au contraire, «[...] quis enim ille sit vere Silvester ac barbarus Sguropulus, si temporum testem historiam, si coaetaneos posterioresque scriptores interroges, homo nihili, ignotus, ignobilis, qui unus sibi testimonium dixit, illudque mendacissimum, neglectus hactenus ab eruditis, schismaticus, haereticus, ac nationis suae, quae catholicam fidem cum imperatore, patriarcha, episcopis, ac principibus doctissimisque viris susceperat, hostis infensissimus illico deprehendetur" (Sacrosancta concilia, ad regiam editionem exacta, quae nunc quarta parte prodit auctior, studio Philip. Labbei et Gabr. Cossartii, Paris, 1672, XIII, col. 1280). 
« Le Pape leur dit qu'ils étoient presentement d'accord, \& qu'il ne restoit plus que quelques petites questions, sur lesquelles il falloit s'éclaircir; qu'elles étoient marquées dans l'écrit qu'il tenoit, \& qu'il leur donna à lire. Il contenoit quatre chefs : Le premier que le Saint Siége \& le Souverain Pontife Vicaire de Jesus-Christ joüiroient de leurs priviléges, \& que l'on reconnoîtroit qu'il avoit pû ajoûter au Symbole ce qu'on y avoit ajoûté ${ }^{24}$. »

L'ajout au Symbole auquel il est fait allusion est évidemment le Filioque, c'est-à-dire le sujet même de la controverse théologique entre les orthodoxes et les Latins. Eugène IV demandait donc aux Byzantins rien moins que de reconnaître la pleine légitimité du pape à intervenir sur le dogme sans concile : or c'est là précisément ce que les orthodoxes reprochaient aux pontifes du $\mathrm{XI}^{\mathrm{e}}$ siècle, qui avaient introduit le Filioque dans le Credo de leur propre initiative. Ce point litigieux avait fait l'objet de plusieurs sessions conciliaires à Ferrare, sans que les Byzantins et les Latins parviennent à un accord. Il ne s'agissait donc pas d'une « petite question », mais d'un différend central, et l'attitude du pape telle qu'elle est narrée apparaît comme une véritable provocation. Du Pin raconte cet épisode en se fondant sur les Actes grecs du concile de Florence et il reste encore une fois très proche de sa source; il rapporte aussi ensuite l'indignation des Grecs face à cette exigence exorbitante ${ }^{25}$. En s'appesantissant sur ce passage, Du Pin exprime peut-être en filigrane ses propres réserves sur la question de l'infaillibilité pontificale.

Sur cette question, il pousse encore plus loin, non pas tant ses développements historiques que l'analyse qu'il propose des œuvres de certains auteurs byzantins. Du Pin consacre quelques pages étonnantes aux traités écrits au XIV siècle par Barlaam de Calabre et par Nil Kabasilas sur la question de la primauté du pape. Non seulement le compte-rendu qu'il en donne implique qu'il les a lus attentivement et qu'il y trouve des idées pertinentes, mais il en fait même ouvertement l'éloge, pour celui de Nil au moins, puisqu'il conclut ainsi :

24. Louis Ellies Du Pin, Histoire des controverses et des matières ecclésiastiques traitées dans le quinziéme siécle, Paris, 1698, p. 162.

25. Voir Quae supersunt Actorum Graecorum Concilii Florentini, éd. Joseph Gill, Rome, 1953 , p. $442^{17-30}$ (demandes du pape) et p. $443^{14-15}$ (réaction des Byzantins). 
« Ces Traitez de Nil sont écrits avec beaucoup d'ordre \& de netteté, $\&$ pleins d'érudition ${ }^{26}$. »

Les deux opuscules étudiés par Du Pin reprennent des arguments à peu près similaires, mais celui de Barlaam inspire à notre théologien des considérations particulièrement intéressantes, qui rejoignent directement sa propre ecclésiologie :

« [Dans son Traité de la Primauté du Pape, Barlaam] soûtient que Jesus-Christ a donné à tous les Apôtres la même puissance de lier $\&$ de délier, qu'il a donnée à Saint Pierre [...]; qu'il n'y a aucune Loi qui oblige les Patriarches de recevoir leur Ordination du Pape; que les Canons ne lui donnent que le premier rang \& la premiere place; qu'il est sujet à l'erreur comme les autres; qu'on ne doit point confondre l'Eglise Romaine avec l'Eglise Catholique, ni faire profession de croire en l'Eglise Romaine comme on croit en l'Eglise Catholique $^{27}$. »

Sont abordés ici les divers aspects du thème de la primauté pontificale : Barlaam, tout comme Du Pin, reconnaît la primauté de Pierre au sein du groupe des apôtres, mais il est beaucoup plus réticent quant à la primauté du siège de Rome, qui n'est qu'honorifique selon les orthodoxes. La notion même d'apostolicité est examinée par Barlaam, qui souligne sa dimension éminemment collégiale, ce qui va tout à fait dans le sens des idées de Du Pin ${ }^{28}$. Dans cette perspective, le successeur de Pierre ne peut se prévaloir d'une quelconque autorité sur les autres patriarches : de fait, la notion de pentarchie est centrale dans l'orthodoxie et elle implique de reconnaître aux cinq patriarcats œcuméniques une dignité égale ${ }^{29}$. Au-delà, c'est l'idée même d'une direction collégiale de l'Église qui est sousentendue, et celle-ci trouve traditionnellement sa meilleure incarnation dans le concile œcuménique. Du Pin ne reprend pas le thème

26. Louis Ellies Du Pin, Histoire des controverses et des matières ecclésiastiques traitées dans le quatorziéme siécle, Paris, 1697, p. 338.

27. Ibid., p. 330-331. L'édition citée par Du Pin est celle de l'érudit protestant Claude Saumaise : Cl. Salmasii Librorum de primatu papae pars prima, cum apparatu. Accessere de eodem primatu Nili et Barlaami tractatus, Amsterdam, 1645 .

28. Voir Jacques Grès-Gayer, « Le gallicanisme de Louis Ellies Du Pin (16571719) », Lias, 18, 1991, p. 47.

29. Sur la question de la primauté, voir François Dvornik, Byzance et la primauté romaine, Paris, 1964 ; John Meyendorff, « St Peter in Byzantine theology », dans The Primacy of Peter. Essays in Ecclesiology and the Early Church, éd. John Meyendorff, Crestwood (NY), 1992, p. 67-90. 
du concile dans le traité de Barlaam $^{30}$; il y fait seulement allusion en creux lorsqu'il évoque la question de l'infaillibilité pontificale : si en effet, comme le pense Du Pin à la suite de Barlaam, le pape est sujet à l'erreur, il n'est pas fondé à prendre seul une décision dogmatique, de sorte qu'une instance supérieure doit être consultée, qui ne peut être que le concile. Cette conception du gouvernement de l'Église rejoint à l'évidence certaines des préoccupations et des revendications des gallicans.

Certaines convergences de vue apparaissent finalement entre le théologien gallican qu'est Du Pin et les auteurs byzantins qu'il étudie. En premier lieu, il faut souligner que Du Pin a lu la majorité des sources grecques auxquelles il se réfère. Les chapitres qu'il consacre à l'histoire byzantine révèlent un érudit dans l'ensemble bien informé et moins approximatif qu'on ne l'a dit parfois; il reste en général fidèle à ses sources, tout en étant capable de les interpréter de façon synthétique pour dresser un tableau suggestif d'une période. Il se montre surtout beaucoup moins prévenu contre les Byzantins que nombre de ses contemporains : sa connaissance des textes grecs les plus variés $-\mathrm{y}$ compris ceux qui ont été publiés par des protestants lui permet de proposer une vision assez juste des événements qu'il relate. L'œuvre de Du Pin témoigne du niveau très honorable de culture byzantine qu'il cherche à transmettre à son lecteur. Mais audelà des informations qu'il compile dans ses ouvrages, Du Pin a aussi beau jeu de reprendre certaines des critiques que les Byzantins formulaient en leur temps à l'encontre de l'Église romaine. Il apparaît ainsi que l'orthodoxie médiévale a maintes raisons d'intéresser les érudits français du XVII ${ }^{\mathrm{e}}$ siècle, tant par sa théologie, fondée sur l'autorité des Pères et des conciles, que par son ecclésiologie.

marie-helene.blanchet@college-de-france.fr

30. Il y figure bien pourtant, mais assez fugitivement: voir la Patrologie grecque, 151, col. 1270. Du Pin cite en revanche Nil Kabasilas sur ce point : « Il montre qu'il n'est pas vrai que le Pape ne puisse être jugé par personne, ni qu'il soit d'un Ordre plus élevé que les Evêques; qu'il est soûmis aux Conciles \& aux Canons; qu'il n'est point à proprement parler Evêque de tout le monde; que le Siege de Rome n'est pas le seul que l'on puisse appeler le Siege Apostolique; qu'il n'appartient pas à lui seul de convoquer le Concile general; \& que si l'on ne peut pas faire de Canons sans lui, il ne peut pas non plus en faire sans les autres » (Louis Ellies Du Pin, Histoire des controverses et des matières ecclésiastiques traitées dans le quatorziéme siécle, Paris, 1697, p. 338). 